\title{
Cerebral Function and Perfusion during Cardiopulmonary Bypass: A Plea for a Multimodal Monitoring Approach
}

\author{
Marcus Thudium, MD, Ingo Heinze, MD, Richard K. Ellerkmann, MD, PhD, Tobias Hilbert, MD \\ Department of Anesthesiology and Intensive Care Medicine, University Hospital Bonn, Germany
}

\section{ABSTRACT}

Background: Postoperative neurological injury still represents a major cause of morbidity after cardiac surgery. Our objective was to compare the limits as well as advantages of routine monitoring tools for the detection of cerebral function and perfusion deficits during cardiopulmonary bypass in a daily clinical setting.

Methods: Adult patients undergoing elective cardiac surgery with use of cardiopulmonary bypass were included. Patients received monitoring comprising Bispectral Index (BIS), Near Infrared Spectroscopy (NIRS) and assessment of middle cerebral artery flow velocity (MCAV) using transcranial Doppler (TCD) sonography. Measurements were taken after anesthesia induction (at baseline) and every 10 minutes during aortic cross-clamping. Relative deviation from baseline values was calculated. Values were compared with predefined, generally accepted threshold values identifying patients at risk for cerebral functional and perfusion deficits.

Results: 30 consecutive patients were included into data analysis. Compared to NIRS as well as BIS monitoring, there was a wide interindividual variability in relative MCAV values for the whole cohort (median 0.9, range 0.39-2.19). Out of 229 measurements in total, 82 BIS but only 30 NIRS and 12 TCD values were lying outside predefined limits. TCD monitoring identified two patients with disturbed cerebral autoregulation, while NIRS remained unremarkable. The

\begin{abstract}
Abbreviations:
POCD postoperative cognitive dysfunction; $r S O 2$ regional cerebral oxygen saturation; NIRS near infrared spectroscopy; ECC extracorporeal circulation; MCAV middle cerebral artery flow velocity; CBF cerebral blood flow; TCD transcranial Doppler ultrasound; EEG electro-encephalography; BIS bispectral index; CPB cardiopulmonary bypass; HLM heart-lung machine; ICU intensive care unit; $\mathrm{CO}$ cardiac output; $\mathrm{Cl}$ cardiac index; MAP mean arterial blood pressure; hgb hemoglobin; CAM-ICU Confusion Assessment Method for the Intensive Care Unit; CABG coronary artery bypass graft;AVR aortic valve replacement; MVR mitral valve replacement;AAR ascending aorta replacement
\end{abstract}

Received August 18, 2017; received in revised form October 26, 2017; accepted November 3, 2017.

Correspondence: Tobias Hilbert, MD, D.E.S.A., Department of Anesthesiology and Intensive Care Medicine, University Hospital Bonn, Sigmund-Freud-Str. 25, D-53127 Bonn, Germany; +49-228-287-14127; fax: +49-228-287-14125 (e-mail: thilbert@uni-bonn.de). latter was significantly associated with systemic hemoglobin levels. Finally, patients with relative MCAV values $>1.0$ had a higher risk of developing postoperative delirium.

Conclusion: Our findings reveal inherent technical limitations of each individual monitoring component, such as high interindividual variability (TCD), low spatial resolution (NIRS), or interaction with anesthetics (BIS). We therefore argue for a multimodal neuromonitoring that combines several qualities. Such approach would help reducing these limitations while individual components complement each other, thus providing more patient safety during cardiac surgery. Furthermore, such an approach would be easily applicable in a routine clinical setting.

\section{INTRODUCTION}

With an aging population, neurological complications following cardiac surgery represent an increasing challenge for the health care systems. They range from deficits such as inadequately waking up after surgery and postoperative delirium to major neurological injury including stroke with severe impairment or even death [Llinas 2000; Newman 2006]. Incidences of neurological deterioration after cardiac surgery vary greatly [Bartels 2013; Llinas 2000]. In previous works, cerebrovascular embolism leading to focal neurological impairment has been separated from injury caused by hypoperfusion, resulting in more generalized complications such as postoperative cognitive dysfunction (POCD) [Bartels 2013; Roach 1996]. Every initiation of actions to prevent or treat serious injury depends on the early detection of adverse events.

Measuring regional cerebral oxygen saturation $\left(\mathrm{rSO}_{2}\right)$ via near infrared spectroscopy (NIRS) has become a widely used tool for intraoperative neuromonitoring during extracorporeal circulation (ECC) in cardiac surgery. It has been validated against much more invasive methods such as jugular bulb oximetry [Daubeney 1996; Murkin 2009]. Cerebral desaturation may occur after cannula malpositioning and can result in POCD or stroke [Colak 2015; Madsen 2000; Zheng 2013]. The role of NIRS in preventing POCD or stroke, however, is disputed, since large randomized controlled trials are missing and reported variability of measured values is high [Bickler 2013; Zheng 2013].

Doppler ultrasound measurement of the middle cerebral artery flow velocity (MCAV) represents cerebral blood flow $(\mathrm{CBF})$, while the method is largely unaffected by vasoactive drugs [Aaslid 1982; Hilz 2000]. Transcranial Doppler (TCD) ultrasound has been used to determine the limits of cerebral autoregulation in combination with NIRS as well as with 
intraoperative electro-encephalography (EEG) to improve cerebral outcome [Hori 2014; Joshi 2012; Zanatta 2011]. Intraoperative EEG, which is used during general anesthesia in the form of processed bispectral index (BIS) monitoring, by itself possesses some predictive value concerning postoperative neurocognitive deterioration \{Chan 2013; Radtke 2013; Soehle 2015].

In many clinical centers, routine cardiac surgery procedures are still done without combined or even single mode neuromonitoring [Erdös 2009]. However, the multifactorial etiology of neurological injury makes it unlikely that all possible threats will be captured effectively using only one single monitoring modality. To evaluate advantages as well as limitations of BIS, NIRS, and TCD monitoring in patients receiving ECC for cardiac surgery, we aimed to record the incidence of deviations from generally accepted threshold values that are considered to indicate intraoperative cerebral functional or perfusion deficits, respectively. Furthermore, individual monitoring modalities were compared to assess their ability to detect such deviations.

\section{METHODS}

This observational study was conducted in accordance with the Declaration of Helsinki and after approval by the institutional revenue board (protocol number 353/16). According to the approval, informed consent was waived due to the pure observational design of the study. Inclusion criteria were: patient age $>18$ years and elective on-pump open heart surgery. The exclusion criteria were as follows: emergency procedure, aortic arch surgery with circulatory arrest, pregnancy, and impossibility to obtain a TCD signal. All patients received anesthesia induction according to standard procedures including arterial line, intubation, central

\section{Patients' Characteristics}

\begin{tabular}{lc}
\hline Age, $y$ & $70(41-82)$ \\
Male sex, $\mathrm{n}(\%)$ & $27(90)$ \\
Weight, $\mathrm{kg}$ & $79(62-119)$ \\
Height, $\mathrm{cm}$ & $176(160-187)$ \\
Body surface area, m2 & $1.99(1.67-2.45)$ \\
Procedure, $\mathrm{n}(\%)$ & \\
CABG & $16(53)$ \\
AVR & $3(10)$ \\
MVR & $4(13)$ \\
AAR & $1(3)$ \\
Combined & $6(20)$ \\
Total & 30 \\
\hline
\end{tabular}

Data are presented as the median (range) unless otherwise indicated. CABG indicates coronary artery bypass graft; AVR, aortic valve replacement; MVR, mitral valve replacement; AAR, ascending aorta replacement. venous catheterization, and urinary catheter. Anesthesia was induced with sufentanil, etomidate, and rocuronium and was maintained with sevoflurane and continuous infusion of sufentanil. The patients received norepinephrine as vasopressor and dobutamine for inotropic support. Median sternotomy was performed in apnea. After systemic heparinization and cannulation of the ascending aorta and of the right atrium or the superior and inferior vena cava, respectively, cardiopulmonary bypass $(\mathrm{CPB}$ ) was established using a roller pump (Advanced Perfusion System 1, Terumo, Tokyo, Japan) and a membrane oxygenator (Quadrox-I Adult, Maquet Getinge Group, Rastatt, Germany). The HLM system was primed with 1200 $\mathrm{mL}$ of crystalloid infusion solution and 10,000 IU of heparin. After full HLM support was installed, ventricular fibrillation was induced, and cardiac arrest was achieved by administering Calafiore warm blood or Bretschneider cardioplegia infusion. The aorta was then cross-clamped. Mild hypothermia (32$34^{\circ} \mathrm{C}$ ) was induced, and acid-base management was performed according to $\mathrm{pH}$-stat regimen. Towards the end of the surgical procedure, patients were rewarmed and subsequently weaned from the $\mathrm{CPB}$ after de-clamping of the aorta and sufficient reperfusion. After successful weaning from the $\mathrm{CPB}$, heparin was antagonized with protamine in an $80-100 \%$ dose of the initially administered heparin. All patients were transferred to the ICU for postoperative care.

Baseline right frontal NIRS (INVOS 5100 Oxymeter, Medtronic, MN, USA), BIS (BIS vista, Medtronic Minimally Invasive Therapies, MN, USA), and TCD measurement of the right MCA was taken after anesthesia induction and before establishing CPB. For TCD, a S5-1 transducer on a

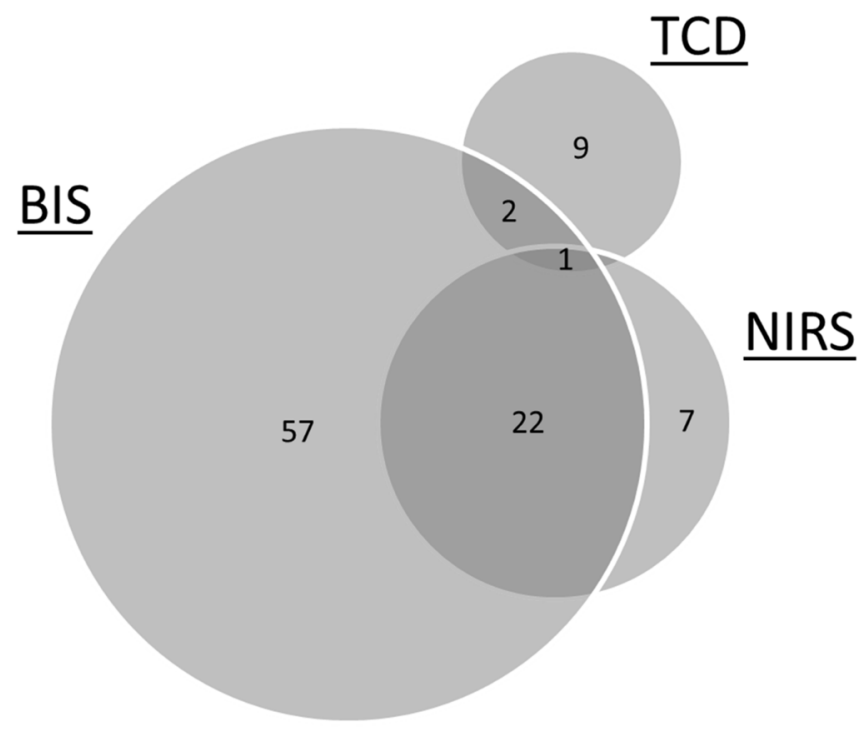

Figure 1. Venn diagram, showing the number of recorded abnormal values, lying outside predefined, established limits for each monitoring modality: TCD, decrease from baseline MCAV by more than $50 \%$ or an increase by more than two-fold or absolute values below $20 \mathrm{~cm} / \mathrm{s}$; NIRS, $>20 \%$ decrease in $\mathrm{rSO} 2$ from baseline values or absolute values below 50\%; BIS, absolute values below 40. TCD indicates transcranial Doppler; BIS, bispectral index, NIRS, near infrared spectroscopy 
CX30 ultrasound system (Philips Medical Systems, Hamburg, Germany) was used. Measurements were performed via a temporal window, as it was previously described by Aaslid et al [Aaslid 1982]. First, the intracranial cavity was visualized in B-mode with a depth of $15 \mathrm{~cm}$, and then the depth was reduced to $8 \mathrm{~cm}$. Color Doppler imaging was added, and the right MCA and the Circle of Willis were visualized. Once an appropriate angle was achieved, pulsed-wave Doppler was added on the M1 segment of the MCA in a depth between 4.5 and $5.5 \mathrm{~cm}$, and averaged blood flow velocity in MCA (MCAV) was measured. After aortic cross-clamping, $\mathrm{rSO}_{2}$,
BIS, and MCAV were monitored every 10 minutes during $\mathrm{CPB}$, and these time points were named $\mathrm{T} 1, \mathrm{~T} 2, \mathrm{~T} 3$. etc. Additional simultaneous data collection included: cardiac output (CO) and cardiac index (CI) from extracorporeal circulation, mean arterial blood pressure (MAP), and hemoglobin (HGB) levels.

According to recent literature, the following established threshold values were defined to identify monitoring results considered abnormal: (1) TCD: decrease from baseline MCAV by more than $50 \%$ or an increase by more than two-fold or absolute values below $20 \mathrm{~cm} / \mathrm{s}$ [Austin 1997]; (2)
A

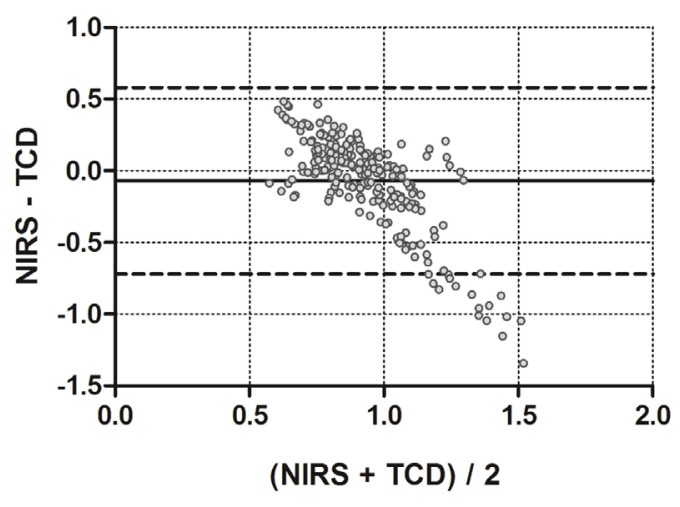

B
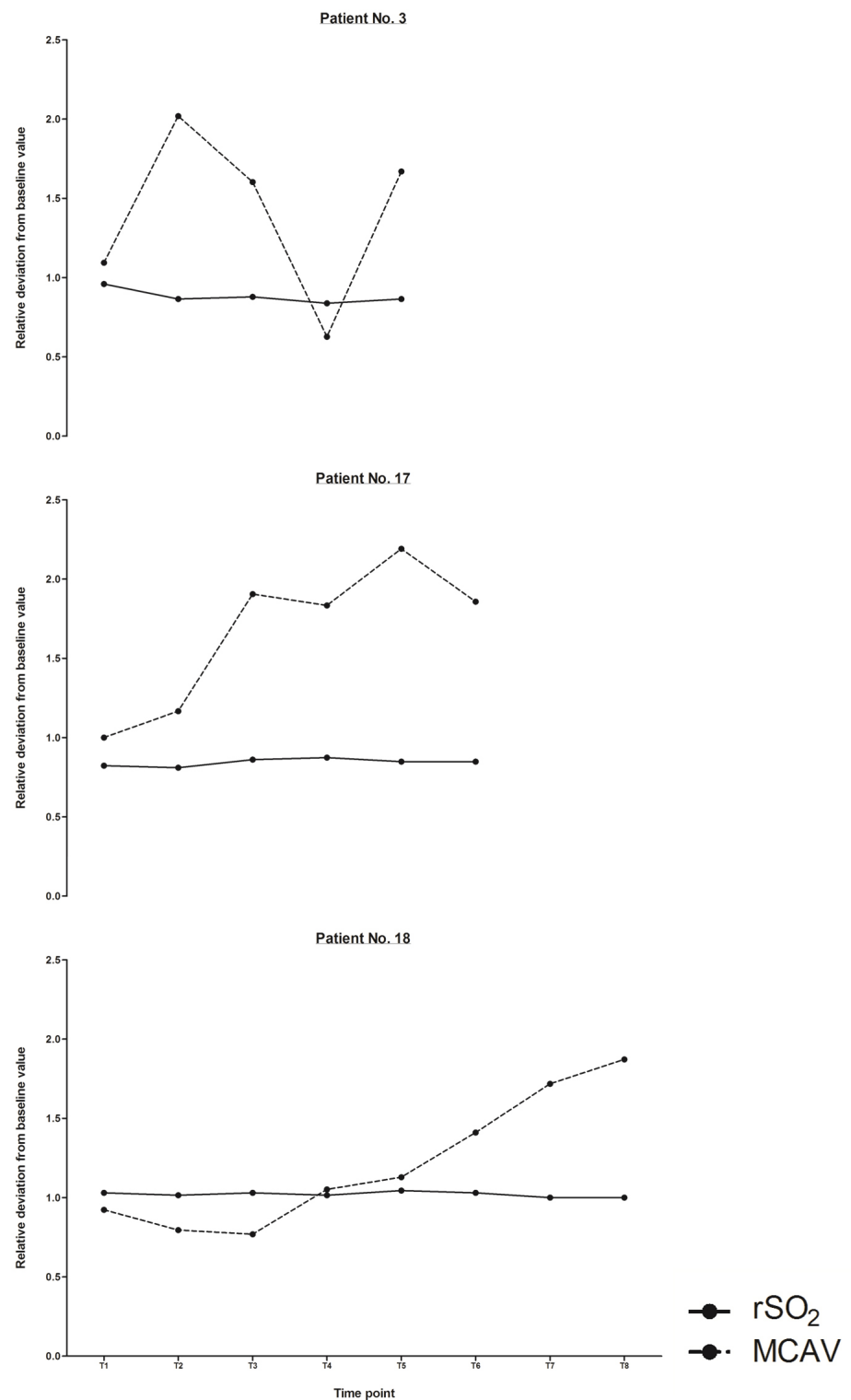

Figure 2. (A) Bland-Altmann Plot comparing NIRS and TCD monitoring (relative deviation from baseline values). The solid black line indicates the mean of differences, and the dashed line indicates the limits of agreement. (B) Individual time course of MCAV and rSO2 values (relative deviation from baseline values) in patients 3, 17, and 18. The solid line shows rSO2 and the dashed line the MCAV values. TCD indicates transcranial Doppler; NIRS, near infrared spectroscopy; $\mathrm{rSO}_{2}$, regional cerebral oxygen saturation; MCAV, middle cerebral artery flow velocity; $\mathrm{T}$, time point of measurement $(\times 10$ minutes after aortic cross-clamping). 


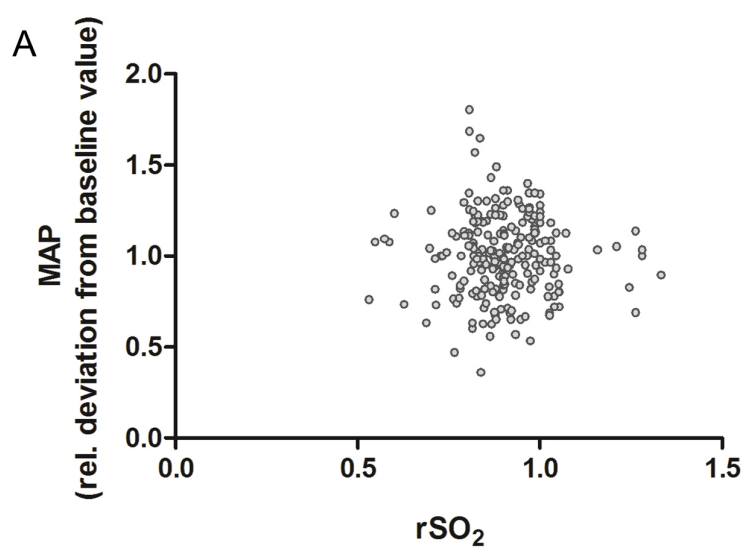

(rel. deviation from baseline value)

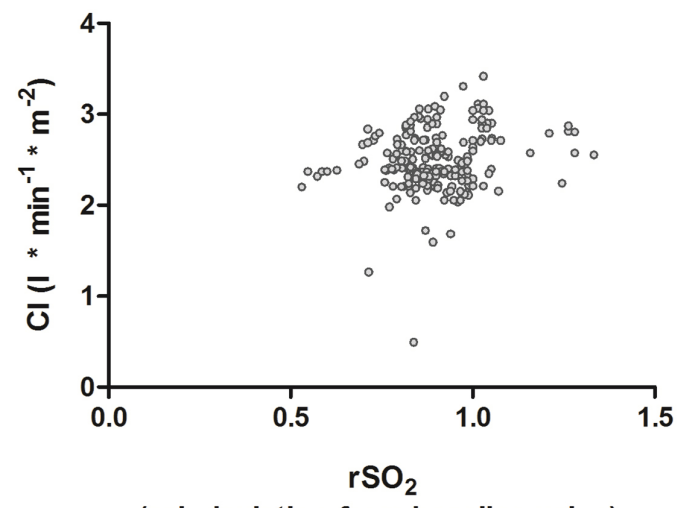

(rel. deviation from baseline value)

B

Patient No. 13

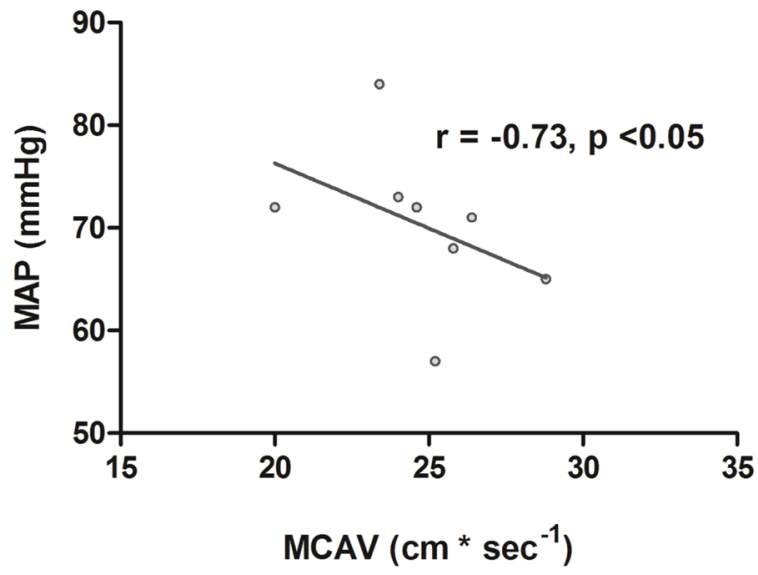

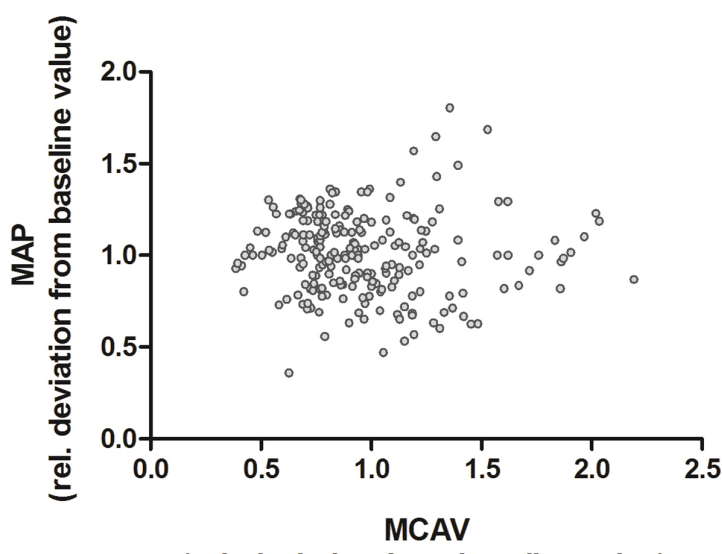

(rel. deviation from baseline value)

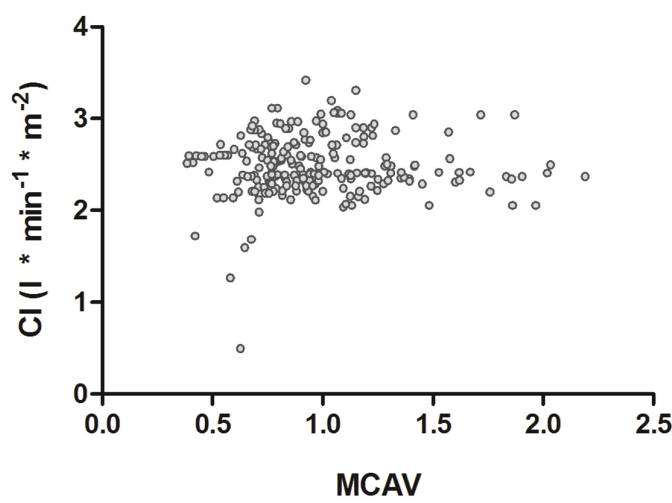

(rel. deviation from baseline value)

Patient No. 23

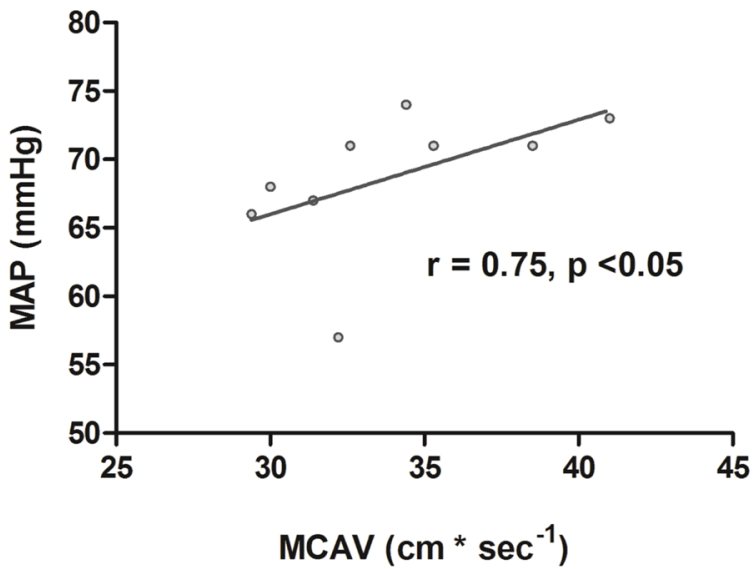

Figure 3. (A) Association between changes in mean arterial blood pressure (MAP) or absolute cardiac index (Cl) with changes in $r \mathrm{SO} 2$ (panels on left side) or in MCAV (panels on right side). MAP, $r \mathrm{SO}_{2}$ and MCAV values are given as relative deviation from baseline values. (B) Association between MAP with $M C A V$ values in two individual patients. MAP and MCAV values are given as absolute values. $\mathrm{SSO}_{2}$ indicates regional cerebral oxygen saturation; $M C A V$, middle cerebral artery flow velocity; $r$, Spearman rank correlation coefficient. 
NIRS: $>20 \%$ decrease in $\mathrm{rSO}_{2}$ from baseline values or absolute values below 50\% [Blas 1999]; (3) BIS: absolute values below 40 [Chan 2013].

Postoperative delirium was assessed in the ICU using the CAM-ICU (Confusion Assessment Method for the Intensive Care Unit) test [Guenther 2010]. Onset of delirium within 24-48 hours after extubation was counted as "postoperative."

All data were transferred into MS Excel (Microsoft, Redmond, WA, USA). Statistical analysis and visualization was performed using GraphPad PRISM 5 (La Jolla, CA, USA). Absolute values were used to analyze individual patients, whereas normalization to baseline was used to analyze the whole cohort. Data are presented as median with range or as individual single values where applicable. Bland-Altman analysis was used to assess method agreement between NIRS and TCD to detect deviations from the predefined limits. Spearman rank correlation coefficient was calculated to assess the association between NIRS- and TCD-derived values and global hemodynamics. $P$ values $<.05$ were considered statistically significant.

\section{RESULTS}

In total, NIRS, BIS, and TCD values from 229 different time points were collected with 32 consecutive patients being monitored and recorded. Two patients were excluded from subsequent analysis, one because of a missing baseline TCD measurement and a second due to the inability to detect a color signal of the MCA during aortic cross-clamping. A median number of 7 time points during clamping was recorded, while the total number of measurements for every individual patient varied due to different lengths of the surgical procedure. For demographics and procedure related data, see the Table. One patient suffered from postoperative low-output syndrome and died on ICU after several days of extracorporeal life support. No patient showed signs of postoperative focal neurological injury, and eight patients (27\%) suffered from postoperative delirium.

Compared to the relative $\mathrm{rSO}_{2}$ (median 0.9 , range 0.53 1.33) or BIS values (median 1.04, range 0.74-1.64), calculated by comparison with the individual baseline, relative MCAV values (median 0.9 ) showed a wide interindividual variability with a range from 0.39-2.19. According to the predefined threshold levels, a large number (82) of recorded BIS values were considered abnormal (Figure 1). In contrast, NIRS returned 30 and TCD 12 values lying outside these predefined limits. NIRS-derived aberrant values showed a considerable overlap with that returned from BIS monitoring.

Despite the Bland-Altman plot for method comparison between NIRS and TCD with a mean of differences of 0.07 indicates a rather limited constant bias between the two methods, the difference between NIRS and TCD-derived values increases with increasing deviation from baseline values (Figure $2 \mathrm{~A}$ ). This is further visualized by Figue $2 \mathrm{~B}$, showing the individual time courses of relative $\mathrm{rSO}_{2}$ and $\mathrm{MCAV}$ values of the three patients with the highest amplitude between the minimum and maximum MCAV. Whereas TCD monitoring indicates a wide, time-dependent variability during $\mathrm{CPB}$, these alterations are not reflected by NIRS.
Concerning our cohort, neither changes in $\mathrm{rSO}_{2}$ nor in measured MCAV values were associated with simultaneous changes in MAP or with absolute CI values measured from ECC (Figure 3A). However, in one patient, absolute MCAV values were associated with MAP values $(\mathrm{r}=0.75, P<.05)$, and in another one, a negative correlation was determined $(\mathrm{r}=-0.73, P<.05)$ (Figure $3 \mathrm{~B})$. Of note, simultaneous NIRS monitoring did not reveal any association with MAP. Analysis of blood samples showed a highly significant correlation between absolute $\mathrm{rSO}_{2}$ values and systemic HGB levels in the whole cohort $(\mathrm{r}=0.46, P<.0001)$.

There was no correlation between values derived from NIRS or BIS monitoring with the development of postoperative delirium. However, 6 of 13 patients $(46 \%)$ that experienced median relative MCAV values $>1.0$ during $\mathrm{CPB}$ developed postoperative delirium, compared to 2 of 17 patients $(12 \%)$ with median relative MCAV values $<1.0$.

\section{DISCUSSION}

Periprocedural neurological injury is still a feared consequence after cardiac surgery and may significantly influence the patient's outcome, eliciting symptoms ranging from minor disorders to major disabilities or even death [Bartels 2013; Roach 1996]. Main mechanisms involved in the development of cerebral disorders are hypoperfusion and embolism. Literature suggests that cerebral function and hemodynamics are too complex to be tackled by one means of monitoring alone [Baussart 2006; Citerio 2015]. The ideal intraoperative neuromonitoring would allow continuous and non-invasive control of brain perfusion and function. Data should easily be interpreted without expert skills, and critical incidents should be detected at an early stage that allows prompt intervention. Our objective was to evaluate routine monitoring tools in a daily clinical setting of cardiac surgery. We demonstrate that parallel neuromonitoring using NIRS, BIS, and TCD is feasible and that such multimodal combination may be advantageous in detecting functional and hemodynamic conditions potentially leading to postoperative neurological deterioration.

We used NIRS to monitor cerebral oxygenation and metabolism. Since this non-invasive method provides continuous information independently of pulsatile blood flow, NIRS has become a standard tool in cardiac surgery during non-pulsatile CPB [Grubhofer 2000]. Its use even in conditions of deep hypothermic circulatory arrest may be beneficial, and protocols have previously been established in order to detect and reverse an actual or impeding cerebral desaturation [Abdul-Khaliq 2001; Denault 2007]. The use of NIRS monitoring has been shown to reduce postoperative neurological complications, although its relevance is still debated [Ghosh 2012; Zheng 2013]. Especially cerebral desaturation was reported to be associated with negative neurological outcome [Colak 2015]. In our study, we used established threshold values of $\mathrm{rSO}_{2}$ to detect cerebral desaturations during ECC in our patients [Blas 1999]. On the one hand, with hemodynamic management based on MAP alone, these desaturations occurred in $>13 \%$ (30 of 229) of the recorded 
time points (Figure 1). On the other hand, NIRS could not indicate considerable changes in cerebral perfusion revealed by TCD monitoring, even in case of a two-fold increase from baseline (Figure 2B). Joshi et al determined the lower limit of cerebral autoregulation using NIRS and demonstrated its wide interindividual variability [Joshi 2012]. Furthermore, this group could show that a blood pressure below these limits was associated with increased morbidity and mortality [Ono 2014]. In our study, there was no significant correlation between low relative MAP values and measured $\mathrm{rSO}_{2}$ values (Figure 3A). Of note, we detected a highly significant association between systemic HGB levels and $\mathrm{rSO}_{2}$. Therefore, altered HGB levels due to surgical blood loss or RBC transfusion may be a relevant confounder of cerebral perfusion monitoring using NIRS on one hand [Torella 2002]. On the other, it has been shown that low HGB levels are associated with adverse cerebral outcome following cardiac surgery, and therefore, NIRS monitoring may be used to guide transfusion strategies [Kulier 2007].

EEG graphically represents the spontaneous cortical electrical activity. Its interpretation usually requires neurophysiological expert skills. A processed EEG, such as the BIS monitoring, can easily be interpreted and has become well established in anesthesiology, not only to monitor drug effects during total intravenous anesthesia [Chan 2013]. We used BIS to monitor the electrophysiological, therefore functional aspects of the brain. Various studies demonstrated that BIS provides a prognostic value for postoperative delirium [Chan 2013; Radtke 2013; Siddiqi 2016; Soehle 2015]. Moreover, under certain conditions, it may allow the detection of cerebral hypoperfusion [Mérat 2001; Sessler 2012]. Of note, the interaction of the EEG signal with anesthetics and hypothermia limits its sensitivity as well as specificity [Billard 2001]. In our series of patients, BIS-derived values fell below established limits $(<40)$ during ECC in a large number of cases $(\mathrm{n}=82)$ (Figure 1) [Chan 2013]. However, there was no association with the development of postoperative neurological or neurocognitive deterioration. The small size of our cohort might limit the detection of a significant clinical influence.

Transcranial Doppler sonography has regained increasing attention due to the availability of more powerful ultrasound systems. Its validity in cardiac surgery has been described in detail [Doblar 2004; Weyland 1994]. Changes in flow velocity in the basal cerebral arteries, assessed through the temporal bone, are supposed to represent changes of $\mathrm{CBF}$, since the arteries' diameter is constant [Hilz 2000]. The administration of vasopressive agents such as norepinephrine exerts no large effect on MCAV [Moppett 2008]. TCD may be of special interest during cardiac surgery, since cerebral autoregulation, ensuring adequate blood flow independent of MAP, can be affected during hypothermic CPB [Joshi 2010]. Instead of a pure Doppler probe, which enables continuous monitoring, we used a regular S5-1 transducer on a common ultrasound device. We did so as the direct view on cerebral vessels adds visual information to the Doppler signal, ensuring adequate signal quality as well as preventing from artifacts due to unnoticed probe dislocation, which otherwise may occur in $>20 \%$ [Moritz 2007]. Moreover, such transducer is usually readily available and no additional device is needed, since ultrasound is used in most patients, e.g. in form of echocardiography or for the installation of intravascular catheters. However, the impossibility of a continuous monitoring in our setting is a limitation, although attaching the transducer to the patient's head is conceivable. Moreover, we experienced difficulties establishing the appropriate acoustic window for proper TCD measurements in one of our patients, a not so uncommon problem [McCarthy 2001]. Despite a reported relationship between NIRS and TCD, Bland-Altman analysis revealed decreasing concordance with increasing deviation from the measured baseline values (Figure 2, A) [Joshi 2012]. In our study, TCD monitoring identified two patients with obviously disturbed cerebral autoregulation, one of which demonstrated even decreasing $\mathrm{CBF}$ with increasing MAP (Figure 3, B). Simultaneous NIRS monitoring did not reveal such association. Of note, individual MAP values in these patients were measured above $60 \mathrm{mmHg}$ and therefore above the commonly reported lower limit of autoregulation [Brady 2010]. Concerning our whole cohort, changing MCAV values were not associated with changes in MAP or with CI values, respectively. This demonstrates that measuring global hemodynamics alone is not sufficient to prevent patients under $\mathrm{CPB}$ from cerebral hypo- or hyperperfusion, as detected by TCD monitoring [Andropoulos 2003].

Combining various monitoring qualities that reflect different aspects is standard in modern anesthesiology and critical care. It seems reasonable that the hemodynamic and metabolic demands of the brain as the most relevant human organ should receive the same attention. Even goal-directed protocols are conceivable, e.g. transfusion algorithms based on cerebral oxygen delivery rather than only hematocrit. Recent review articles likewise stress the indispensability of composite approaches towards intraoperative monitoring due to the multifactorial and complex causes of neurological injury [Guarracino; 2008; Mittnacht 2014]. Moritz et al compared various methods to monitor cerebral perfusion deficits during awake carotid endarterectomy, which allowed intraoperative communication with the patient and thus the exact detection of neurologic deterioration [Moritz 2007]. The authors were able to derive receiver operating characteristic curves for each of the investigated monitoring modalities. They concluded that "none of the monitoring systems under investigation provided perfect sensitivity and specificity." Combining different techniques would be the logical consequence. Due to the small size of our cohort, we are not able to reliably assess effects of our regimen on clinical outcome. However, we noticed that the incidence of postoperative delirium was greater among patients who experienced median relative MCAV values $>1.0$ during $\mathrm{CPB}$, indicating that relative cerebral hyperperfusion might play a significant role.

In summary, our findings reveal inherent physiological limitations of each individual monitoring component, such as high interindividual variability (TCD), low spatial resolution (NIRS), or interaction with anesthetics (BIS). Cerebral autoregulation may be impaired during cardiopulmonary bypass, calling for a more individualized hemodynamic management. We therefore argue for multimodal neuromonitoring that 
integrates various qualities and therefore may adequately cover important aspects of cerebral oxygenation, hemodynamics, and electrophysiology. Such an approach would help to reduce these limitations while individual components complement each other, thus providing more patient safety. Furthermore, such an approach would be easily applicable in a routine clinical setting. The relevance of our findings on parameters of clinical outcome remains to be investigated. Since monitoring alone does not improve patients' outcome, prospective studies establishing intervention protocols are urgently needed.

\section{REFERENCES}

Aaslid R, Markwalder TM, Nornes H. 1982. Noninvasive transcranial Doppler ultrasound recording of flow velocity in basal cerebral arteries. J Neurosurg 57:769-74.

Abdul-Khaliq H, Schubert S, Troitzsch D, et al. 2001. Dynamic changes in cerebral oxygenation related to deep hypothermia and circulatory arrest evaluated by near-infrared spectroscopy. Acta Anaesthesiol Scand 45:696-701.

Andropoulos DB, Stayer SA, McKenzie ED, Fraser CD. 2003. Novel cerebral physiologic monitoring to guide low-flow cerebral perfusion during neonatal aortic arch reconstruction. J Thorac Cardiovasc Surg 125:491-9.

Austin EH, Edmonds HL, Auden SM, et al. 1997. Benefit of neurophysiologic monitoring for pediatric cardiac surgery. J Thorac Cardiovasc Surg 114:707-15, 717-16.

Bartels K, McDonagh DL, Newman MF, Mathew JP. 2013. Neurocognitive outcomes after cardiac surgery. Curr Opin Anaesthesiol 26:91-7.

Baussart B, Cheisson G, Compain M, et al. 2006. Multimodal cerebral monitoring and decompressive surgery for the treatment of severe bacterial meningitis with increased intracranial pressure. Acta Anaesthesiol Scand 50:762-5.

Bickler PE, Feiner JR, Rollins MD. 2013. Factors affecting the performance of 5 cerebral oximeters during hypoxia in healthy volunteers. Anesth Analg 117:813-23.

Billard V. Brain injury under general anesthesia: is monitoring of the EEG helpful? 2001. J Can Anesth 48:1055-60.

Blas M, Sulek C, Martin T, Lobato E. 1999. Use of near-infrared spectroscopy to monitor cerebral oxygenation during coronary artery bypass surgery in a patient with bilateral internal carotid artery occlusion. J Cardiothorac Vasc Anesth 13:732-5.

Brady K, Joshi B, Zweifel C, et al. 2010. Real-time continuous monitoring of cerebral blood flow autoregulation using near-infrared spectroscopy in patients undergoing cardiopulmonary bypass. Stroke 41:1951-6.

Chan MT, Cheng BC, Lee TM, et al. 2013. BIS-guided anesthesia decreases postoperative delirium and cognitive decline. J Neurosurg Anesthesiol 25:33-42.

Citerio G, Oddo M, Taccone FS. 2015. Recommendations for the use of multimodal monitoring in the neurointensive care unit: Curr Opin Crit Care 21:113-19.

Colak Z, Borojevic M, Bogovic A, Ivancan V, Biocina B, Majeric-Kogler V. 2015. Influence of intraoperative cerebral oximetry monitoring on neurocognitive function after coronary artery bypass surgery: a randomized, prospective study. Eur J Cardio-Thorac Surg Off J Eur Assoc Cardio-Thorac Surg 47:447-54.
Daubeney PE, Pilkington SN, Janke E, Charlton GA, Smith DC, Webber SA. 1996. Cerebral oxygenation measured by near-infrared spectroscopy: comparison with jugular bulb oximetry. Ann Thorac Surg 61:930-4.

Denault A, Deschamps A, Murkin JM. 2007. A proposed algorithm for the intraoperative use of cerebral near-infrared spectroscopy. Semin Cardiothorac Vasc Anesth 11:274-81.

Doblar DD. 2004. Intraoperative transcranial ultrasonic monitoring for cardiac and vascular surgery. In: Seminars in Cardiothoracic and Vascular Anesthesia. Vol 8. SAGE Publications; 127-45. http://scv.sagepub.com/ content/8/2/127.short. Accessed April 12, 2017.

Erdös G, Tzanova I, Schirmer U, Ender J. 2009. Neuromonitoring and neuroprotection in cardiac anaesthesia. Nationwide survey conducted by the Cardiac Anaesthesia Working Group of the German Society of Anaesthesiology and Intensive Care Medicine]. Anaesthesist 58:247-58.

Ghosh A, Elwell C, Smith M. 2012. Review article: cerebral near-infrared spectroscopy in adults: a work in progress. Anesth Analg 115:1373-83.

Grubhofer G, Mares P, Rajek A, et al. 2000. Pulsatility does not change cerebral oxygenation during cardiopulmonary bypass. Acta Anaesthesiol Scand 44:586-91.

Guarracino F. 2008. Cerebral monitoring during cardiovascular surgery. Curr Opin Anaesthesiol 21:50-4.

Guenther U, Popp J, Koecher L, et al. 2010. Validity and reliability of the CAM-ICU Flowsheet to diagnose delirium in surgical ICU patients. J Crit Care 25:144-51.

Hilz MJ, Stemper B, Heckmann JG, Neundörfer B. 2000. Mechanisms of cerebral autoregulation, assessment and interpretation by means of transcranial doppler sonography. Fortschr Neurol Psychiatr 68:398-412.

Hori D, Brown C, Ono M, et al. 2014. Arterial pressure above the upper cerebral autoregulation limit during cardiopulmonary bypass is associated with postoperative delirium. Br J Anaesth 113:1009-17.

Joshi B, Brady K, Lee J, et al. 2010. Impaired autoregulation of cerebral blood flow during rewarming from hypothermic cardiopulmonary bypass and its potential association with stroke. Anesth Analg 110:321-8.

Joshi B, Ono M, Brown C, et al. 2012. Predicting the limits of cerebral autoregulation during cardiopulmonary bypass. Anesth Analg 114:503-10.

Kulier A, Levin J, Moser R, et al. 2007. Impact of preoperative anemia on outcome in patients undergoing coronary artery bypass graft surgery. Circulation 116:471-9.

Llinas R, Barbut D, Caplan LR. 2000. Neurologic complications of cardiac surgery. Prog Cardiovasc Dis 43:101-12.

Madsen PL, Secher NH. 2000. Postoperative confusion preceded by decreased frontal lobe haemoglobin oxygen saturation. Anaesth Intensive Care 28:308-10.

McCarthy RJ, McCabe AE, Walker R, Horrocks M. 2001. The value of transcranial doppler in predicting cerebral ischaemia during carotid endarterectomy. Eur J Vasc Endovasc Surg 21:408-12.

Mérat S, Lévecque JP, Le Gulluche Y, Diraison Y, Brinquin L, Hoffmann JJ. 2001. BIS monitoring may allow the detection of severe cerebral ischemia]. J Can Anesth 48:1066-9.

Mittnacht AJC, Rodriguez-Diaz C. 2014. Multimodal neuromonitoring in pediatric cardiac anesthesia. Ann Card Anaesth 17:25.

Moppett IK, Sherman RW, Wild MJ, Latter JA, Mahajan RP. 2008. Effects of norepinephrine and glyceryl trinitrate on cerebral haemodynamics: transcranial Doppler study in healthy volunteers. Br J Anaesth 100:240-4. 
Moritz S, Kasprzak P, Arlt M, Taeger K, Metz C. 2007. Accuracy of cerebral monitoring in detecting cerebral ischemia during carotid endarterectomy: a comparison of transcranial Doppler sonography, near-infrared spectroscopy, stump pressure, and somatosensory evoked potentials. Anesthesiology 107:563-9.

Murkin JM, Arango M. 2009. Near-infrared spectroscopy as an index of brain and tissue oxygenation. Br J Anaesth 103(Supplement 1):i3-i13.

Newman MF, Mathew JP, Grocott HP, et al. 2006. Central nervous system injury associated with cardiac surgery. The Lancet 368:694-703.

Ono M, Brady K, Easley RB, et al. 2014. Duration and magnitude of blood pressure below cerebral autoregulation threshold during cardiopulmonary bypass is associated with major morbidity and operative mortality. J Thorac Cardiovasc Surg 147:483-9.

Radtke FM, Franck M, Lendner J, Krüger S, Wernecke KD, Spies CD. 2013. Monitoring depth of anaesthesia in a randomized trial decreases the rate of postoperative delirium but not postoperative cognitive dysfunction. Br J Anaesth 110 Suppl 1:i98-105.

Roach GW, Kanchuger M, Mangano CM, et al. 1996. Adverse cerebral outcomes after coronary bypass surgery. Multicenter Study of Perioperative Ischemia Research Group and the Ischemia Research and Education Foundation Investigators. N Engl J Med 335:1857-63.

Sessler DI, Sigl JC, Kelley SD, et al. 2012. Hospital stay and mortality are increased in patients having a "triple low" of low blood pressure, low bispectral index, and low minimum alveolar concentration of volatile anesthesia. Anesthesiology 116:1195-203.

Siddiqi N, Harrison JK, Clegg A, et al. 2016. Interventions for preventing delirium in hospitalised non-ICU patients. In: The Cochrane Collaboration, ed. Cochrane Database of Systematic Reviews. Chichester, UK: John Wiley \& Sons, Ltd. http://doi.wiley.com/10.1002/14651858. CD005563.pub3. Accessed December 2, 2016.

Soehle M, Dittmann A, Ellerkmann RK, Baumgarten G, Putensen C, Guenther U. 2015. Intraoperative burst suppression is associated with postoperative delirium following cardiac surgery: a prospective, observational study. BMC Anesthesiol 15:61.

Torella F, Cowley R, Thorniley MS, McCollum CN. 2002. Monitoring blood loss with near infrared spectroscopy. Comp Biochem Physiol A Mol Integr Physiol 132:199-203.

Weyland A, Stephan H, Kazmaier S, et al. 1994. Flow velocity measurements as an index of cerebral blood flow. Validity of transcranial Doppler sonographic monitoring during cardiac surgery. Anesthesiology 81:1401-10.

Zanatta P, Messerotti Benvenuti S, Bosco E, Baldanzi F, Palomba D, Valfrè C. 2011. Multimodal brain monitoring reduces major neurologic complications in cardiac surgery. J Cardiothorac Vasc Anesth 25:1076-85.

Zheng F, Sheinberg R, Yee M-S, Ono M, Zheng Y, Hogue CW. 2013. Cerebral near-infrared spectroscopy monitoring and neurologic outcomes in adult cardiac surgery patients: a systematic review. Anesth Analg 116:663-76. 